\title{
RELAÇÃO ENTRE SATISFAÇÃO E EFICIÊNCIA RELATIVA: uma aplicação no segmento da hospitalidade hoteleira em Belém-PA.
}

\author{
Américo Johny Jaques Melo \\ Universidade da Amazônia (UNAMA) \\ Av. Alcindo Cacela, 287 Umarizal - Belém-PA- Brasil \\ americojaques@hotmail.com \\ Sergio Castro Gomes \\ Universidade da Amazônia (UNAMA) \\ Av. Alcindo Cacela, 287 Umarizal - Belém-PA- Brasil \\ sergio.gomes@unama.com
}

\section{RESUMO}

O objetivo deste estudo é calcular e analisar, a partir da Análise Envoltória de Dados DEA, o nível da eficiência relativa no setor hoteleiro e realizar uma comparação com o indicie de satisfação, por meio do modelo de regressão linear. Utilizou-se como parâmetro de dados informações coletadas em 18 hotéis na região metropolitana de Belém. Com isso, aplicou-se a ferramenta SIAD v3 que calculou a eficiência dos processos desses hotéis, utilizando cinco variáveis, nas quais são três de input: o ano de existência do hotel, o número de empregados e números de quartos e duas variáveis output: o número médio de quartos ocupados e RevPar (Receita por Quarto Disponível), como bases importantes para análise. Empregou-se o modelo $\mathrm{BCC}$, orientado por output, para a demonstração dos resultados obtidos em quatro grupos: o da eficiência padrão, o da eficiência na fronteira invertida, o da eficiência composta e o da eficiência composta normalizada. Os resultados revelaram que apenas um dos hotéis apresentou eficiência $100 \%$ e a comparação entre a eficiência calculada e o índice de satisfação obteve uma tendência crescente, no qual quando mais eficiente a organização, maior seu índice de satisfação.

Palavra-chave: Eficiência, DEA, Setor Hoteleiro, regressão linear.

\section{ABSTRACT}

The objective of this study is to calculate and analyze, from the exchange rate analysis, the level of benefit related to the hotel sector and to make a comparison with the satisfaction index, using the linear regression model. It was used as a parameter of information measurements collected in 18 hotels in the metropolitan region of Belém. With this, we can use a SIAD v3 tool that calculates the efficiency of the hotel data, which are five variables, which are three of them. Input: The year of existence of the hotel, the number of customers and the number of rooms and two output variables: the average number of occupied rooms and RevPar (Revenue per Available Room), as important bases for analysis. The outputoriented BCC model was used to demonstrate the results obtained in four groups: standard efficiency, inverted efficiency, composite efficiency and normalized distribution. The results revealed that only one minute had a rising trend, and an index between satisfaction and 
satisfaction index of a growing offer, when the company was efficient and the satisfaction index.

Keywords: Efficiency, DEA, Hotel Sector, linear regression.

\section{Como Citar:}

MELO, Américo; GOMES, Sergio. RELAÇÃO ENTRE SATISFAÇÃO E EFICIÊNCIA RELATIVA: uma aplicação no segmento da hospitalidade hoteleira em Belém-PA. In: SIMPÓSIO DE PESQUISA OPERACIONAL E LOGÍSTICA DA MARINHA, 19., 2019, Rio de Janeiro, RJ. Anais do SPOLM 2019. Rio de Janeiro: Centro de Análises de Sistemas Navais, 2019.

\section{INTRODUÇÃO}

Atualmente os hotéis são os meios de hospedagem comuns encontrados. A hotelaria é um ramo de comercio que tem como foco o turismo de modo geral e tem como objetivo trabalhar nas áreas de hospedagem, alimentação e outras atividades relacionadas ao bem-estar dos clientes, prezando sempre pela qualidade e satisfação dos clientes.

O turismo é reconhecido universalmente como um setor marcante a nível econômico, cultural, social e ambiental. Em 2017, a indústria de viagens e turismo continua a fazer uma verdadeira diferença para a vida de milhões de pessoas no mundo, impulsionando o crescimento, criando empregos, reduzindo a pobreza e promovendo o desenvolvimento e a tolerância. Pelo sexto ano consecutivo, o crescimento da indústria supera o da economia global, mostrando a resiliência da indústria diante da incerteza geopolítica global e da volatilidade econômica (SCHWAB et al., 2017).

A performance dos hotéis no Brasil em 2017 foi marcada pelo terceiro ano consecutivo da queda do REVPAR. A pequena recuperação da economia do país permitiu que a ocupação média subisse de 55,2\% em 2016 para 56,5\% em 2017, no entanto a diária média caiu 6,7\% em 2017 comparado com o ano anterior (JLL HOTELS \& HOSPITALITY GROUPS, 2017). O desempenho dos hotéis começou o ano de 2018, apresentando a tendência de melhora dos seus fundamentos iniciada no segundo semestre de 2017. No entanto, vários fatores externos como o aumento da taxa de juros nos Estados Unidos e que acabaram gerando uma fuga de capital externo do país e consequentemente a desvalorização da moeda local (Real), começou a impactar significativamente a economia do país (JLL HOTELS \& HOSPITALITY GROUPS, 2017).

Nos dias atuais, frente a um mercado cada vez mais competitivo e exigente, as organizações precisam possuir métodos de gestão mais precisos. No ramo hoteleiro não é diferente, devido à inserção de grandes redes de hotéis internacionais, a competitividade nesse setor está maior (SILVA; MARTINS, 2017).

Agora que a competitividade entre os hotéis está aumentando, os gerentes de hotéis estão começando a perceber que melhorar seu desempenho pode se tornar sua vantagem e com analise gerencial essas melhorias podem ser identificadas e feitas (Min \& Min, 1997). Com o nivel de competitividade maior no mercado hotelerio nacional, se espera que as organizações busquem novas estratégias para melhorar seus serviços prestados, com o objetivo de conquistar um maior percentual de mercado para si e satifazer seus clientes.

Com esse crescimento na buscar de melhorar os processos empresarias e suas tomadas de decisão, muitas empresas buscam forma de redução dos seus custos para trabalhar de formar mais eficiente possível. Avaliar a eficiência é de fundamental importância porque auxilia no processo decisório e oferece subsídios que facilitam o monitoramento, a comparação e a 
correção do desempenho organizacional (DOUMPOS; COHEN, 2014). Sendo assim, a medida da eficiência torna-se um assunto importante e abrangente.

Sendo assim, pode se dizer que, com a competitividade e a busca pela eficiência crescendo em relação ao setor hoteleiro, a necessidade e a procura por técnicas que irão auxiliar na tomada de decisão. Neste fato, aponta-se a Análise Envoltória de Dados (DEA) como ferramenta que auxilia na tomada de decisão.

Portanto, essa pesquisa pode ser sintetizada na seguinte questão: Qual o nível de eficiência dos hotéis e sua relação com a satisfação do cliente na região metropolitana de Belém-PA? No qual, o DEA será utilizado no presente trabalho com objetivo de calcular o nível da eficiência técnica e a análise de restrição com o objetivo de comparar a eficiência com a satisfação do cliente das unidades hoteleiras.

\section{REFERENCIAL TEÓRICO}

\subsection{EFICIÊNCIA NA HOTELARIA}

Segundo Chen, Liang, Yang e Zhu (2006), as medidas de eficiência podem fornecer aos gestores hoteleiros uma análise adicional no sentido da melhoria do uso dos seus recursos, desempenhando também um papel crucial na rentabilidade e sobrevivência das empresas. Portanto, faz-se necessário a utilização de métodos que auxiliem os gestores desse setor para que as organizações obtenham uma boa competitividade no mercado.

Segundo Marques e Silva (2006), a eficiência de uma organização ou atividade/processo refere-se à comparação entre os valores dos insumos (inputs) e os produtos/resultados (outputs) e os seus valores ótimos. No contexto da hotelaria, os aspetos da eficiência se relacionam com a satisfação do cliente e a análise de indicadores de desempenho.

Tais indicadores de desempenho oferecem informações importantes e essências para benchmarking com base no desempenho contábil e financeiro, porém há muitos fatores em relação ao desempenho do hotel, e, obviamente, esses indicadores não levam em consideração a mistura e a natureza dos serviços prestados. Como sugerido por Anderson et al. (1999), medir a eficiência relativa de um hotel requer métodos que são mais sensíveis e contábeis e que podem considerar explicitamente vários insumos e saídas do hotel.

\subsection{APLICAÇÃo dA ANÁliSE ENVOLTÓRIA DE DADOS (DEA) NO SETOR HOTELEIRO}

Em 1996, Parkan aplicou várias técnicas para avaliar o desempenho das empresas hoteleiras nos EUA. Uma das técnicas foi DEA. Esta foi uma das primeiras tentativas de medir a eficiência das empresas hoteleiras aplicando este método.

Em 2015, foi avaliado hotéis de 4 e 5 estrelas em Portugal, com objetivo de analisar a eficiência, porém a analise considerou dois modelos distintos: num são utilizados variáveis com unidades em quantidades e no outro com unidades monetárias (Oliveira et at., 2015). Souza et at. (2016), utilizaram o DEA para analisar a eficiência em relação a sustentabilidade nos hotéis-fazendas na Região Agreste de Pernambuco.

De acordo com Poldrugovac et al. (2016), os estudos na área da indústria hoteleira usaram vários recursos contábeis financeiros e informação não financeira para variáveis de entrada e saída. Neste trabalho antes de definir as variáveis de entrada (input) e saída (output), foi analisado aquelas usadas pelos autores na revisão da bibliografia. Foram selecionados os autores, o ano de publicação e as variáveis de entrada (input) e saída (output), demonstrando todo essa informação na Tabela 1: 
Tabela 1 - Revisão de bibliografia das variáveis de entrada (input) e saídas (output) mais frequentes.

\begin{tabular}{|c|c|c|}
\hline Autores & INPUT & OUTPUT \\
\hline Tumer (2010) & $\begin{array}{l}\text { Capacidade do quarto (1) } \\
\text { Custo do pessoal (2) } \\
\text { Custo de energia (3) } \\
\text { Custo F \& B (4) } \\
\text { Outro custo (5) }\end{array}$ & $\begin{array}{l}\text { RevPAR modificado (1) } \\
\text { Outras receitas por quarto vendido (2) }\end{array}$ \\
\hline $\mathrm{Wu}$, et at. (2010) & $\begin{array}{l}\mathrm{N}^{\mathrm{o}} \text { de quartos }(1) \\
\mathrm{N}^{\mathrm{o}} \text { de empregados }(2) \\
\text { Capacidade do F\&B (3) } \\
\text { Total dos custos operacionais (4) }\end{array}$ & $\begin{array}{l}\text { Receita do alojamento (1) } \\
\text { Receita do F\&B (2) } \\
\text { Outras receitas (3) }\end{array}$ \\
\hline Manasakis, et at. (2012) & $\begin{array}{l}\mathrm{N}^{\mathrm{o}} \text { Empregados (1) } \\
\mathrm{N}^{\mathrm{o}} \text { Quartos (2) } \\
\text { Custo operacional (3) }\end{array}$ & $\begin{array}{l}\text { Receitas totais (1) } \\
\text { Overnight (2) }\end{array}$ \\
\hline Rebelo, et at. (2013) & $\begin{array}{l}\mathrm{N}^{\mathrm{o}} \text { de empregados (1) } \\
\text { Ativo fixo líquido (2) } \\
\text { Gastos operacionais (3) }\end{array}$ & Vendas totais líquidas (1) \\
\hline Ashrafi, et at. (2013) & $\begin{array}{l}\text { Taxa de quarto média padrão (1) } \\
\text { Chegadas internacionais (2) } \\
\text { PIB (3) }\end{array}$ & $\begin{array}{l}\text { Receita de quarto de hotel (1) } \\
\text { Receita de bebidas e comida (2) } \\
\text { Taxa de ocupação ( } 3 \text { ) } \\
\text { Outras receitas (4) }\end{array}$ \\
\hline Oliveira, et at. (2015) & $\begin{array}{l}\mathrm{N}^{\mathrm{o}} \text { quartos }(1) \\
\mathrm{N}^{\mathrm{o}} \text { funcionários }(2) \\
\text { Capacidade } \mathrm{F} \& \mathrm{~B}(3) \\
\text { Outros custos (4) } \\
\text { Custos com o pessoal (5) } \\
\text { Custos de capital (6) }\end{array}$ & Receita Total (1) \\
\hline Souza, et at. (2016) & $\begin{array}{l}\text { Número total de funcionários (1) } \\
\text { Porcentagem de funcionários da } \\
\text { localidade (2) } \\
\text { Número de leitos (3) } \\
\text { Consumo médio de energia (4) } \\
\text { Consumo médio da água (5) } \\
\text { Re-úso médio da água (6) } \\
\text { Incorporação dos requisitos } \\
\text { ambientais nos produtos e serviços (7) }\end{array}$ & $\begin{array}{l}\text { Valor da diária com taxas (1) } \\
\text { Porcentagem da taxa de ocupação média } \\
\text { (2) } \\
\text { Índices de satisfação dos clientes (3) } \\
\text { Porcentagem de resultados financeiros } \\
\text { decorrentes de ações sustentáveis (4) }\end{array}$ \\
\hline $\begin{array}{l}\text { Poldrugovac, et at. } \\
\text { (2016) }\end{array}$ & $\begin{array}{l}\text { Despesas de energia (1) } \\
\text { Despesas de sala (2) } \\
\text { Despesas de F \& B (3) } \\
\text { Despesas associadas a outros serviços } \\
\text { (4) } \\
\text { Despesas trabalhistas (5) }\end{array}$ & $\begin{array}{l}\text { Rendimento total (1) } \\
\text { Taxa de ocupação (2) }\end{array}$ \\
\hline
\end{tabular}

Fonte: Resultados da pesquisa.

De acordo com a Tabela, nota-se que as variáveis mais utilizadas em relação a inputs foram número de quartos e números de funcionários, no caso dos outputs foram as receitas e taxa de ocupação, entretanto, das variáveis que mais se relaciona com o ramo de serviço foi o índice de satisfação do cliente. Com o avanço da tecnologia de informação do aparecimento dos sites de gestão de hospedagem como Booking, Tripadvisor e outros que apresentam o nível de satisfação como medida do desempenho sendo uma informação crucial para os gestores. 


\subsection{ANÁLISE ENVOLTÓRIA DE DADOS (DEA)}

A Analise Envoltória de Dados (do inglês Data Envelopment Analysis - DEA) é considerada como uma ferramenta matemática utilizada para medir a eficiência de unidades produtivas, DMU's (MELLO et al., 2003).

Segundo Freitas et al. (2017) essa técnica possibilita que o desempenho relativo de unidades designadas por DMU's (Decision Marking Units) seja analisado. DMU's são unidades que utilizam os mesmos tipos de insumos para a produção dos mesmos bens e/ou serviços. Insumos e produtos esse que podem ser variáveis continuas, ordinais ou categóricas e podem ser medidas em diferentes unidades (ANDRÉ et al., 2018).

Há vários modelos para o DEA, o que deu início ao modelo foi o CRS (Constant Returns to Scale), que é apropriado quando todas as DMU's estão funcionando em uma mesma escala ótima, se for o contrário, as variações de escala afetam a eficiência técnica (VARELA, 2008). Enquanto o modelo BCC (VRS - Variable Returns of Scale), originado por Banker, Charnes e Cooper em 1984, é uma extensão do modelo anterior com acréscimo da restrição, em que ela apontará comparações entre a DMU não eficiente com a eficiente se elas forem de tamanhos similares (VARELA, 2008).

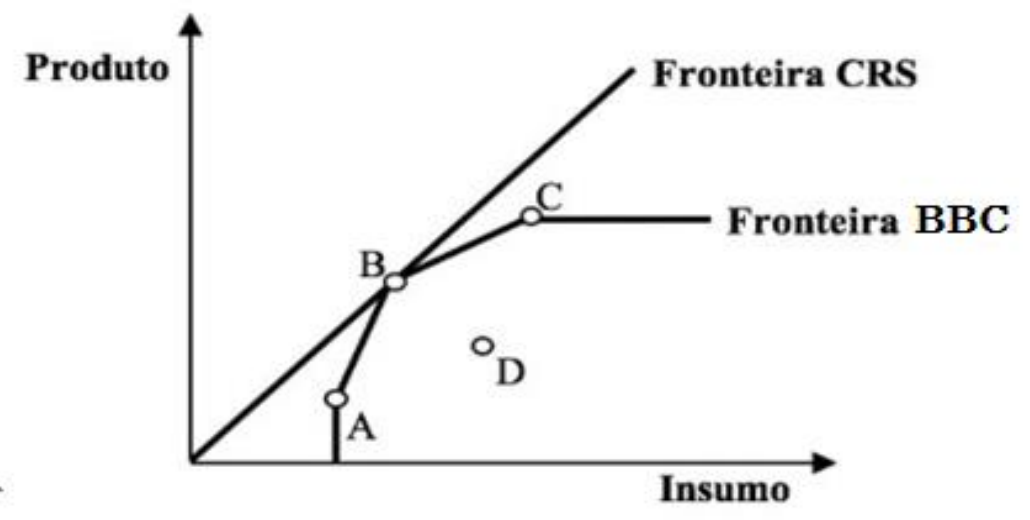

Figura 01: Fronteiras de eficiência de modelos do DEA CRS e BBC.

Fonte: Adaptada de Macedo et al. (2015, p. 58).

Os modelos de DEA podem ser orientados a input, quando se almeja a adaptação da eficiência por meio da diminuição de insumos, tendo constante o nível de produção, ou a output, que, de forma semelhante, é utilizado quando se deseja o ajustamento do nível de eficiência por meio do aumento da produção, preservando constante o nível de insumos.

Neste trabalho, será utilizado o modelo BCC, para a mensuração da eficiência técnica no setor hoteleiro, pois esse modelo trabalha com uma variação de escala e não atribui parida entre input e output, sendo ótimo na indicação da eficiência técnica. Além do mais, será aplicado à fronteira invertida para melhor aproveitamento dos dados.

\subsection{MODELO BCC}

O modelo BCC, abreviatura de Banker, Charnes e Cooper, que o desenvolveram e apresentaram em artigo publicado na Management Science em 1984. O Modelo BCC pressupõe que as unidades avaliadas apresentem retornos variáveis de escala. Segundo BELLONI (2000, p. 68) "ao possibilitar que a tecnologia exiba propriedades de retornos à escala diferentes ao longo de sua fronteira, esse modelo admite que a produtividade máxima varie em função da escala de produção". A formulação matemática do Modelo BCC, com orientação ao output, é: 


$$
\begin{gathered}
h_{o}=\sum_{i=1}^{r} v_{i} x_{i o}+v^{*} \\
\text { Sujeito a } \\
\sum_{j=1}^{s} u_{j} y_{j o}=1 \\
-\sum_{i=1}^{r} v_{i} x_{i k}-\sum_{j=1}^{s} u_{j} y_{j k}-v * \leq 0, k=1, \ldots, s \\
u_{j}, v_{i} \geq 0 \quad \forall x, y \\
u^{*} \in \Re
\end{gathered}
$$

A orientação da análise por output, isto é, tem como objetivo manter os insumos na mesma amplitude e melhorar os produtos (output). Logo após a coleta de dados, estes foram organizados em plataforma Excel, e será utilizado o Sistema de Apoio à Decisão - SIAD v3 para a realização do cálculo de eficiência.

\subsection{FRONTEIRA INVERTIDA}

Quando se obtém resultados onde existem muitas unidades produtivas que alcançam a fronteira de eficiência torna-se difícil a escolha pelo administrador da melhor unidade produtiva. Para distingui-las é necessário introduzir o conceito de fronteira invertida, que consiste em considerar os produtos como insumos e os insumos como produtos. Esse enfoque considera pelo menos duas interpretações. A primeira é que a fronteira consiste das unidades produtivas com as piores práticas gerenciais (e poderia ser chamada de fronteira ineficiente); a segunda é que essas mesmas unidades produtivas têm as melhores práticas considerando o ponto de vista oposto (MELLO et al., 2003).

Para o cálculo da fronteira de ineficiência é feita uma troca dos inputs com os outputs do modelo DEA original. A Figura 2 mostra as duas fronteiras, a clássica e a invertida, para o caso DEA BCC.

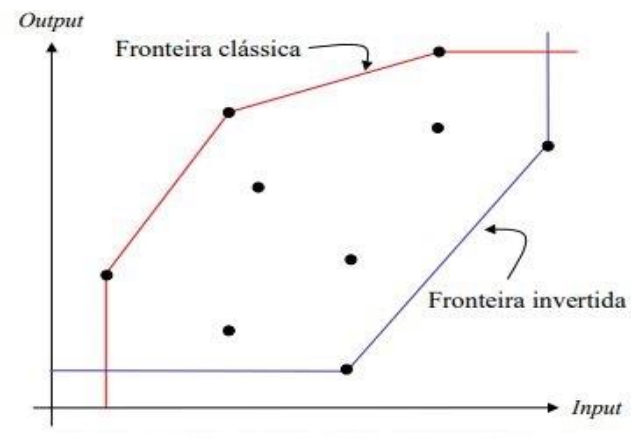

Figura 2: Mostra um exemplo de uma fronteira clássica comparada com uma fronteira invertida.

Fonte: Soares de Mello et al. (2005). 
Após análise das unidades de tomada de decisão e do conjunto de variáveis, pode-se combinar a fronteira padrão e a invertida e obter-se os seguintes tipos de eficiência: eficiência composta, obtida a partir da combinação da eficiência clássica com a invertida, conforme a equação 3 .

$$
\text { Eficiência Composta }=\frac{\text { Eficiência Padrão+(1-Eficiência Invertida })}{2}
$$

E a eficiência composta normalizada obtida a partir da divisão do valor da eficiência composta pelo maior valor entre todos os valores de eficiência composta, conforme definido por Angulo Meza et al. (2005) e expressa na equação 4.

$$
\text { Eficiência Composta Normalizada }=\frac{\text { Eficiência Composta }}{\text { Max(Eficiência Composta })}
$$

\section{METODOLOGIA}

A pesquisa segue uma aplicação de métodos quantitativos, quando do levantamento empírico realizado junto a um conjunto de 18 hotéis, a partir da aplicação da técnica de multicasos, sendo seis de rede internacional e os demais independentes, e aplicando o método matemático e não-paramétrico de Análise Envoltória de Dados (DEA). Considerando as variáveis de acordo com a tabela 1, definiram-se 3 variáveis input: o número de empregados, números de quartos e anos de existência e 2 variáveis output: RevPar (Receita por Quarto Disponível) e número médio de quartos ocupados. Além disso, foi realizado uma comparação entre eficiência e índice de satisfação, no qual teve como base as informações do site Tripadvisor, utilizando o modelo de regressão linear.

Os hotéis pesquisados estão localizados na Região Metropolitana de Belém (RMB) e fazem parte do Sindicato dos Hotéis e Restaurantes de Belém e Ananindeua (SHORES) com o qual a Universidade da Amazônia tem convênio de cooperação técnico-científica para estudos sobre práticas de gestão na hotelaria paraense. O presente projeto amplia a produção científica desenvolvida pelos pesquisadores do Núcleo de Estudos de Práticas de Gestão, Desempenho e Competitividade das Organizações na Amazônia - NEGOA.

\section{RESULTADO E DISCUSSÃO}

\subsection{MEDIDAS DESCRITIVAS}

A tabela 2 demonstrar as principais medidas estatísticas das variáveis utilizadas no modelo BCC orientado por input.

Tabela 2 - Análise exploratória de dados

\begin{tabular}{ccccc}
\hline Variável & Média & Desvio padrão & Mínimo & Máximo \\
\hline Anos de existência & 11,94 & 12,85 & 1,00 & 45,00 \\
$\mathrm{~N}^{\circ}$ de empregados & 48,72 & 40,09 & 7,00 & 190,00 \\
$\mathrm{~N}^{\circ}$ de quartos & 153,44 & 99,04 & 9,00 & 405,00 \\
$\mathrm{~N}^{\circ}$ médio de quartos ocupados & 72,39 & 48,68 & 3,00 & 211,00 \\
RevPar (Receita por Quarto & 90,52 & 48,21 & 32,62 & 197,32 \\
Disponível) & & &
\end{tabular}


Fonte: Resultados da pesquisa.

Para a realização do cálculo e analise da eficiência dos hotéis, por meio do DEA, foram utilizadas 3 variáveis de input, sendo qual os anos de existência do hotel, número de empregados e o número de quartos, para as variáveis de output, foram o número médio de quartos ocupados e o RevPar (Receita por Quarto Disponível). O estudo para a escolha das variáveis foi com base a Tabela 1 , em relação as variáveis mais utilizadas considerando a acessibilidade das mesmas.

\subsection{APLICAÇÃO DA METODOLOGIA DEA NO SETOR HOTELEIRO}

Analisando a Tabela 3, dos resultados obtidos por meio do software SIAD v3, percebese pelos valores da fronteira padrão que as DMUs H5, H6, H9, H11, H13, H15 E H5 são eficientes apresentando valores igual a 1. As DMUs mais ineficientes foram as $\mathrm{H} 3$ e H1, demonstrando valores próximos de zero. Os resultados demonstrados pela fronteira invertida afirmam que a DMU H13 é ineficiente se contradizendo ao resultado calculados pela fronteira padrão, obtendo uma falsa eficiência.

Por razões de confiabilidade, os hotéis são nomeados por números e é apresentado seus tipos de gestão, no qual 8 são de rede e 10 são independentes.

Tabela 3 - Medidas de Eficiência padrão, invertida e composta, modelo BCC.

\begin{tabular}{cccccc}
\hline DMU & TIPO DE & PADR $\tilde{O} \mathbf{0}$ & $\begin{array}{c}\text { EF. } \\
\text { INVERTIDA }\end{array}$ & $\begin{array}{c}\text { EF. } \\
\text { COMPOSTA }\end{array}$ & $\begin{array}{c}\text { EF. COMPOSTA } \\
\text { NORMALIZADA }\end{array}$ \\
\hline H1 & Idenpedente & 0,4902 & 0,9537 & 0,2682 & 0,3079 \\
H2 & Idenpedente & 0,6754 & 0,5844 & 0,5455 & 0,6262 \\
H3 & Rede & 0,3027 & 1,0000 & 0,1513 & 0,1737 \\
H4 & Idenpedente & 0,6006 & 1,0000 & 0,3003 & 0,3447 \\
H5 & Idenpedente & 1,0000 & 0,6921 & 0,6540 & 0,7507 \\
H6 & Rede & 1,0000 & 0,2578 & 0,8711 & 1,0000 \\
H7 & Rede & 0,6291 & 1,0000 & 0,3146 & 0,3611 \\
H8 & Rede & 0,5479 & 0,5008 & 0,5235 & 0,6010 \\
H9 & Rede & 1,0000 & 0,2665 & 0,8668 & 0,9950 \\
H10 & Idenpedente & 0,7414 & 0,3846 & 0,6784 & 0,7788 \\
H11 & Rede & 1,0000 & 0,5290 & 0,7355 & 0,8443 \\
H12 & Rede & 0,8729 & 1,0000 & 0,4364 & 0,5010 \\
H13 & Rede & 1,0000 & 1,0000 & 0,5000 & 0,5740 \\
H14 & Idenpedente & 1,0000 & 0,3851 & 0,8074 & 0,9269 \\
H15 & Idenpedente & 1,0000 & 0,4084 & 0,7958 & 0,9136 \\
H16 & Idenpedente & 0,8770 & 0,5124 & 0,6823 & 0,7832 \\
H17 & Idenpedente & 0,7619 & 0,7669 & 0,4975 & 0,5712 \\
H18 & Idenpedente & 1,0000 & 1,0000 & 0,5000 & 0,5740 \\
\hline Média & & 0,8055 & 0,6801 & 0,5627 & 0,6460 \\
Desvio & & 0,2206 & 0,2856 & 0,2147 & 0,2465 \\
Padrão & & & & & \\
\hline Fon: & & & & \\
\hline
\end{tabular}

Fonte: Resultados da pesquisa.

$\mathrm{Na}$ Tabela 4 os hotéis foram classificados de acordo com a sua eficiência por intermédio da fronteira composta e a fronteira composta normalizada, proporcionando uma melhor avaliação das DMUs e demonstrando um ranking para uma melhor visualização. Além disso, 
é indicado para cada hotel seu índice de satisfação, de acordo com as informações do site Tripadvisor. Portanto, foram retratados os valores percentuais de cada DMU, com isso possibilitou analisar que dentre os hotéis a mais eficiente é a DMU H6 e o que apresentou a menor eficiência dentre todas foi a DMU H3 gerando uma eficiência de 17,37\%.

Tabela 4 - Ranking da eficiência composta normalizada das empresas.

\begin{tabular}{|c|c|c|c|}
\hline POSIÇÃO & DMU & $\begin{array}{l}\text { EF. COMPOSTA } \\
\text { NORMALIZADA }\end{array}$ & IND. SATISF. \\
\hline $1^{\mathrm{o}}$ & H6 & $100,00 \%$ & 8 \\
\hline $2^{\circ}$ & H9 & $99,50 \%$ & 8 \\
\hline $3^{\circ}$ & H14 & $92,69 \%$ & 8,9 \\
\hline $4^{\circ}$ & H15 & $91,36 \%$ & 8,5 \\
\hline $5^{\circ}$ & H11 & $84,43 \%$ & 7,5 \\
\hline $6^{\circ}$ & H16 & $78,32 \%$ & 8,1 \\
\hline $7^{\circ}$ & H10 & $77,88 \%$ & 8,1 \\
\hline $8^{\circ}$ & H5 & $75,07 \%$ & 7,1 \\
\hline $9^{\circ}$ & $\mathrm{H} 2$ & $62,62 \%$ & 8 \\
\hline $10^{\circ}$ & $\mathrm{H} 8$ & $60,10 \%$ & 8 \\
\hline $12^{\circ}$ & H18 & $57,40 \%$ & 7,7 \\
\hline $11^{\circ}$ & H13 & $57,40 \%$ & 7,5 \\
\hline $13^{\circ}$ & H17 & $57,12 \%$ & 7,6 \\
\hline $14^{\circ}$ & H12 & $50,10 \%$ & 7,3 \\
\hline $15^{\circ}$ & $\mathrm{H7}$ & $36,11 \%$ & 7,4 \\
\hline $16^{\circ}$ & $\mathrm{H} 4$ & $34,47 \%$ & 7,4 \\
\hline $17^{\circ}$ & $\mathrm{H} 1$ & $30,79 \%$ & 6,9 \\
\hline $18^{\circ}$ & $\mathrm{H} 3$ & $17,37 \%$ & 7,5 \\
\hline
\end{tabular}

Fonte: Resultados da pesquisa.

\subsection{COMPARAÇÃO COM EFICIÊNCIA E SATISFAÇÃO DOS CLIENTES}

Além desses resultados obtidos pelo DEA, o estudo analisou qual é a relação entre eficiência e o índice de satisfação dos clientes, verificando qual é o grau de relação entre essas duas variáveis, eficiência e satisfação.

Tabela 5 - Resumo do modela de regressão linear

\begin{tabular}{|c|c|c|c|c|}
\hline Modelo & $\mathrm{R}$ & $\mathrm{R}$ quadrado & $\begin{array}{c}\text { R quadrado } \\
\text { ajustado }\end{array}$ & $\begin{array}{c}\text { Erro padrão } \\
\text { da estimativa }\end{array}$ \\
\hline 1 &, $659^{\mathrm{a}}$ &, 434 &, 397 &, 39711 \\
\hline
\end{tabular}

a. Preditores: (Constante), VAR00001

Fonte: Resultados da pesquisa.

Percebe-se, a partir da Tabela 5 de resumo do modelo de regressão linear, que o coeficiente de explicação (R quadrado) corresponde ao valor de 0,434 , ou seja, este resultado indica que 43,4\% da variabilidade da satisfação do cliente é explicada pelo modelo estimado que inclui a variável explicativa eficiência. Desta mesma tabela, percebe-se que existe 
correlação positiva entre eficiência dos hotéis e satisfação dos clientes, ao nível de significância de $1 \%$.

Tabela 6 - Validade do modelo estimado

\begin{tabular}{|c|c|c|c|c|c|c|}
\hline \multicolumn{1}{|c|}{ Modelo } & $\begin{array}{c}\text { Soma dos } \\
\text { Quadrados }\end{array}$ & $\mathrm{gl}$ & $\begin{array}{c}\text { Quadrado } \\
\text { Médio }\end{array}$ & $\mathrm{F}$ & Sig. \\
\hline \multirow{3}{*}{1} & Regressão & 1,817 & 1 & 1,817 & 11,522 &, $004^{\mathrm{b}}$ \\
& Resíduo & 2,365 & 15 &, 158 & & \\
& Total & 4,182 & 16 & & & \\
\hline
\end{tabular}

a. Variável Dependente: VAR00002

b. Preditores: (Constante), VAR00001

Fonte: Resultados da pesquisa.

Outro resultado importante encontrado a partir da Tabela 6 diz respeito à validade do modelo estimado (regressão), pois, conforme o quadro de Análise de Variância (ANOVA), há a indicação de que modelo estimado que representa a explicação da satisfação do cliente em função da eficiência dos hotéis é altamente significativo a $1 \%$.

Tabela 7 - Coeficientes lineares dos modelos

\begin{tabular}{|c|c|c|c|c|c|}
\hline \multirow{2}{*}{ Modelo } & \multicolumn{2}{|l|}{ Coeficientes não padronizados } & $\begin{array}{c}\text { Coeficientes } \\
\text { padronizados }\end{array}$ & \multirow{2}{*}{$\mathrm{t}$} & \multirow{2}{*}{ Sig. } \\
\cline { 2 - 5 } & $\mathrm{B}$ & Erro Padrão & Beta & &, 000 \\
\hline 1 (Constante) & 6,893 &, 271 & & 25,421 &, 004 \\
\hline
\end{tabular}

a. Variável Dependente: VAR00002

Fonte: Resultados da pesquisa.

Com base no Tabela 7 de coeficientes lineares, percebe-se que tanto o intercepto (constante) quanto o coeficiente linear da eficiência dos hotéis são altamente significativos a $1 \%$. Deste modo, infere-se que independente da eficiência do hotel, a satisfação média dos cientes é em torno de 6,893; e que para cada unidade de eficiência alcançada pelo hotel, há um acréscimo médio de 0,013 pontos na satisfação do ciente, o que é bastante considerável. Isto indica, por exemplo, que para um hotel que consegue aumentar em $10 \%$ a sua eficiência, o mesmo produzirá, em média, um aumento de 1,3 pontos na satisfação (medido numa escala de 0 a 10) do cliente, ao nível de significância de $1 \%$.

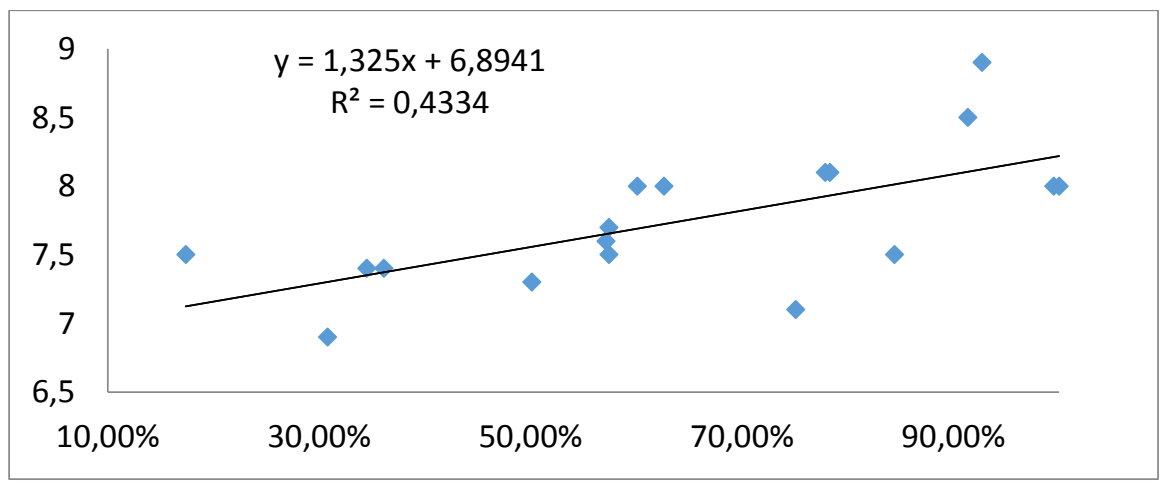

Figura 3:Gráfico de dispersão

Fonte: Resultados da pesquisa. 
As conclusões anteriores podem ser confirmadas com base na Figura 3, onde é possível verificar uma tendência crescente nas coordenadas de pontos que relacionam a satisfação dos clientes com a eficiência dos hotéis.

\section{CONCLUSÃO}

Considerando que o setor do turismo tem um papel importante no reajuste da estrutura produtiva nacional, este estudo teve como finalidade de realizar um estudo empírico sobre a comparação entre eficiência e satisfação do setor hoteleiro da região metropolitana de Belém, no estado do Pará Com isso, aplicou-se a modelagem Analise Envoltória de Dados - DEA, por ser uma metodologia de análise importante para melhoria de empresas, tendo como importância no apoio ao processo de tomada de decisão dos gestores, além disso, foi realizado uma comparação pelo modelo de regressão linear entre satisfação do cliente e o nível de eficiência relativa.

A análise da avaliação da eficiência no setor hoteleiro, da região metropolitana de Belém, foi desenvolvida pelo modelo BCC, no qual foram pesquisados 18 hotéis com tipo de gestão diferente. Os resultados demonstraram pelo modelo de regressão linear apresentaram uma forte relação entre o indicie de satisfação dos clientes, no qual foi obtido do site Tripadvisor, e a eficiência relativa.

Esta analise pode ajudar os empresários e gestores do ramo hoteleiro na tomada de decisão e na busca de novas estratégias para melhorar seu índice de eficiência e, consequentemente, seu nível de satisfação com seus clientes.

Portanto, com a utilização desse método, pode-se favoreceu para novos incentivos no setor hoteleiro, no qual possam incentivar no desenvolvimento do Estado. Para novos estudos, sugere-se a aplicação do método orientado para input, para que estes ou outros hotéis atingirem novos conhecimentos sobre o que estão gerando como serviço tornam suas estratégias eficientes e ajudem a sobreviverem no mercado, sendo mais competitivos. 


\section{REFERÊNCIAS}

ANDERSON, R., FISH, M., XIA, Y., MICHELLO, E. "Measuring Efficiency in the Hotel Industry: A Stochastic Frontier Approach", International Journal of Hospitality Management, 18, p.45-57, 1999.

ANDRÉ, B. S.; RODRIGO, R. R. F.; WELLINGTON, G. Mapeamento e análise bibliométrica da utilização da Análise Envoltória de Dados (DEA) em estudos de engenharia de produção. Brazilian Journal of Production Engineering, v, 4 n. 1, 2018.

ANGULO MEZA, L.; BIONDI NETO, L.; MELLO, J. C. C. B. S.; GOMES, E, G, ISYDSIntegrated System for Decision Support (SIAD - Sistema Integrado de Apoio a Decisão): a software package for data envelopment analysis model, Pesquisa Operacional, v,25, n,3, p 493-503, 2005.

ASHRAFI, A., SEOWB, H. V., LEE, L. S., LEE, C. G.. The efficiency of the hotel industry in Singapore. Tourism Management, 37, 31-34, 2013.

BELLONI, J., A. Uma metodologia de avaliação da eficiência produtiva de Universidade Federais Brasileiras. 2000. Tese (Doutorado em Engenharia de Produção) -Programa de PósGraduação em Engenharia de Produção, do Departamento de Engenharia de Produção e Sistemas, da Universidade Federal de Santa Catarina. Florianápolis, 2000.

CHEN, Y., LIANG, L., YANG, F., ZHU, J. Evaluation of information technology investment: a data envelopment analysis approach. ComputersOperational Research, 33(5), 1368-1379, 2006. doi: 10.1016/j.cor.2004.09.021

DOUMPOs, M.; COHEN, S. Applying data envelopment analysis on accounting data to assess and optimize the efficiency of Greek local governments. Omega International Journal of Management Science, v. 46, p. 74- 85, 2014.

FREITAS, A. P.; GUTERRES, M. X.; LAMPERT, V. N.; SILVAS, A. H. S.; BARCELLOS, J. O. J. Aplicação de metodos de seleção de variáveis em um modelo DEA na produção de bovinos de corte. Engevista, v. 19, n. 4, p. 881-889, 2017.

JLL HOTELS \& HOSPITALITY GROUPS. Hotelaria em números-Brasil 2017. 2017. MACEDO, F. F. R.; KLOPPPEL; N. R.; RODRIGUES JÚNIOR; M. M.; Scarpin; J.E. Análise da eficiência dos recursos públicos direcionados à educação: estudo nos municípios do Paraná. Administração Pública e Gestão Social; v.7, n.2, 2015.

MANASAKIS, C., APOSTOLAKIS, A., \& DATSERIS, G.. Using data envelopment analysis to measure hotel efficiency in Crete. International Journal of Contemporary Hospitality Management, 25, 510-535, 2012.

MARQUES, R., \& SILVA, D. (2006). Inferência estatística dos estimadores de eficiência obtidos com a técnica fronteira não paramétrica de DEA: uma metodologia de Bootstrap. Investigação Operacional, 26(1), 89-110.

MELLO, J. C. C. B. S. et al. Análise de envoltória de dados no estudo da eficiência e dos benchmarks para companhias aéreas brasileiras, Pesquisa Operacional, v, 23, n, 2, p, 324$345,2003$.

MELlo, J. C. C. B. S.; MEZA, L. A., GOMES, E. G., NETO, L. B. Curso de análise de envoltória de dados. In: Simpósio Brasileiro de Pesquisa Operacional, 37, 2005, Gramado. Anais... Rio de Janeiro: SOBRAPO, 2005. 
MEZA, L. A., BIONDI NETO, L., MELLO, J., GOMES, E., COELHO, P. FSDA-FREE software for decision analysis (slad-software livre de apoio à decisão): a software package for data envelopment analysis models. in Congreso Latino-iberoamericano de Investigación Operativa-CLAIO, volume 12, 2004.

MIN, H., MIN, H. Benchmarking the quality of hotel services: managerial perspectives.The. International Journal of Quality \& Reliability Management, 14, 582-597, 1997.

OLIVEIRA, R. S. L. P., PEDRO M. I. C. e MARQUES, R. D. R. C.. Avaliação da Eficiência das Empresas Hoteleiras do Algarve pela Metodologi Análise Envoltória de Dados (DEA). Revista Brasileira de Gestão de Negócios, vol. 17, 788-805 , 2015.

POLDRUGOVAC, K., TEKAVCIC, M., JANKOVIC, S.. Efficiency in the hotel industry: an empirical examination of the most influential factors, Economic Research- Ekonomska Istraživanja, 29:1, 583-597, 2016.

REBELO, S., MATIAS, F., CARRASCO, P. Aplicação da metodologia DEA na análise da eficiência do setor hoteleiro português: uma análise aplicada às regiões portuguesas Tourism \& Management Studies, Vol. 9, 21-28, 2013.

SCHWAB, K.; MARTIN, C.; SAMANS, R.; MOAVENZADEH, J.; DRZENIEK-HANOUZ, M. The Travel \& Tourism Competitiveness Report 2017. World Economic Forum, 2017.

SILVA, F. K.; MARTINS, V. A. A Utilização da Contabilidade Gerencial no Ramo Hoteleiro no Município de Foz do Iguaçu-PR. Revista Eletrônica Ciências da Administração e Turismo. Vol. 5, Núm. 2, Páginas 40 - 54, 2017.

SOUZA, V., MELO, A. S., SOBRAL, M.F. F., VALENÇA, M. N.. Uso da análise envoltória de dados para mensuração da sustentabilidade de hotéis-fazenda em Pernambuco. Interações (Revista internacional de desenvolvimento local), v. 18, 41-57, 2016.

TUMER, N.. Measuring Hotel Performance Using Data Envelopment Analysis, Anatolia: An International Journal of Tourism and Hospitality Research, 21:2, 271-287, 2010.

VARELA, P. S. Financiamento e controladoria dos municípios paulistas no setor saúde: uma avaliação de eficiência. Tese Doutorado, Universidade de São Paulo. São Paulo, 2008. WU, Jie, Liang, Liang \& SONG, Haiyan.. Measuring hotel performance using the integer DEA model. Tourism Economics, 16(4), 867-882, 2010. 